\title{
Programación multiobjetivo de quirófanos considerando el bienestar del cliente interno y externo
}

\author{
Milton A. Londoño, Cristiam A. Gil, Juan S. Mock kow y Juan P. Orejuela * \\ Escuela de Ingeniería Industrial, Universidad del Valle, Cali -Colombia. \\ (correo-e: milton.londono@correounivalle.edu.co; cristiam.gil@correounivalle.edu.co; \\ Juan.mock@correounivalle.edu.co; juan.orejuela@correounivalle.edu.co ) \\ ${ }^{*}$ Autor a quien debe ser dirigida la correspondencia
}

Recibido May. 12, 2021; Aceptado Jul. 12, 2021; Versión final Sep. 20, 2021, Publicado Feb. 2022

\begin{abstract}
Resumen
Este estudio tiene como propósito presentar un modelo multiobjetivo para el problema de programación de quirófanos, que sincroniza las necesidades de los clientes externos con los recursos disponibles y al mismo tiempo los requerimientos de los clientes internos. Se emplea la programación por compromisos buscando la eficiencia de los siguientes objetivos: (1) maximización de la preferencia total, (2) minimización de los tiempos ociosos y (3) maximización del número de clientes. La función de preferencia propuesta considera para cada cirugía, la prioridad dada por el médico, su duración y fecha máxima de asignación, logrando atender la urgencia de la cirugía y disminuir el tiempo de espera. El modelo se valida con un caso real en Colombia, y los resultados demuestran un desempeño por encima del 89.9\% de cercanía al óptimo ideal en cada uno de los objetivos propuestos. Se concluye que el uso de herramientas multiobjetivo para problemas de programación de cirugías produce buenos resultados en todas las medidas de desempeño consideradas.
\end{abstract}

\section{Multi-objective operating room scheduling considering the well-being of internal and external clients}

\begin{abstract}
The purpose of this study is to present a multi-objective model for scheduling operating room problems by synchronizing the needs of external clients with available resources while meeting the requirements of internal clients. A compromise scheduling is applied to improve: (1) efficiency of total preference maximization, (2) idle time minimization, and (3) maximization of client number. The proposed preference function considers surgery priorities set by doctors, which include assigned period and maximum scheduling lateness. This manages the urgency of each surgery and reduces waiting time. The model is validated with a real case in Colombia. The results show a performance above $89.9 \%$ of the ideal optimum for each of the proposed objectives. It is concluded that by using multi-objective tools to solve operating room scheduling problems provides satisfactory results for each of the performance parameters considered.
\end{abstract}




\section{INTRODUCCIÓN}

Un componente importante dentro de los hospitales es la actividad quirúrgica, se estima que esta genera alrededor de dos tercios de los ingresos y cerca del $40 \%$ de los costos en recursos, que incluyen costos de personal e instalaciones (Conforti et al., 2010). La programación de cirugías hace parte de una de las actividades que se abordan dentro de la logística hospitalaria; en esta actividad se deben definir la sincronización de tiempos de inicio y finalización de intervenciones entre los pacientes y recursos disponibles: cirujanos, enfermeras, anestesistas, salas quirúrgicas entre otros (Akbarzadeh et al., 2019). En los últimos años, se estima que la demanda de servicios quirúrgicos aumenta debido al creciente número de afiliaciones a los sistemas de salud y al incremento de la expectativa de vida lo cual genera una mayor necesidad de atención médica por cada década más de vida y triplica los costos del sistema (Velásquez-Restrepo et al., 2013).

Por otro lado, las cancelaciones de cirugías, están relacionadas con la inapropiada programación, asignación de recursos y planificación de la atención al paciente (Ministerio de Salud,2015) tal como lo plantea TorresSanchez y Estupiñan-Gonzalez (2014), que concluyen que las principales causas para la cancelación de cirugías son: Políticas y métodos de programación inadecuados, desconocimiento de horarios de programación, retrasos en el ingreso y preparación del paciente y retrasos o ausencias de insumos. Una inadecuada planeación y programación de cirugías conlleva a usar horas extras de recursos limitados como las salas y los equipos médicos (Médicos, enfermeras, anestesiólogos), además implica demoras en la cirugía o cancelación de los procedimientos, que son costosos tanto para los pacientes como para el hospital (Conforti et al., 2010). Es por esta razón que los centros asistenciales deben de optimizar al máximo la utilización de los recursos que disponen para atender la creciente demanda de servicios quirúrgicos (Velásquez-Restrepo et al., 2013).

Sin embargo, a la hora de optimizar los recursos quirúrgicos por medio de una apropiada planeación y programación de cirugías existen diferentes actores, con diferentes motivaciones, lo cual genera conflictos de interés al materializarse sus respetivas preferencias. Por ejemplo, un administrador del hospital estaría interesado en lograr niveles de eficiencia altos y bajos costos, mientras que el personal médico podría preocuparse por laborar menos horas extras y obtener un mejor bienestar, en cambio al paciente, podría importarle poco los factores mencionados anteriormente y desear tiempos de espera cortos (Samudra et al., 2016). En este tipo de problemas donde todos los actores juegan un papel relevante en el proceso decisorio e inciden en el éxito de la organización, la optimización multi objetivo a diferencia de la mono objetivo, brinda la posibilidad de encontrar soluciones eficientes y balanceadas que permiten una mirada más integral al problema y a su naturaleza.

Existe toda una serie de técnicas formales soportadas en la investigación de operaciones las cuales han sido desarrolladas en el contexto del mismo problema o que son adoptadas de entornos similares en otras industrias. Entre los métodos de solución derivados de la investigación de operaciones, los más utilizados en la literatura para el problema de programación de cirugías son los métodos exactos. Sin embargo, existe un número importante de trabajos realizados con métodos aproximados y simulación (Zhu et al., 2019). Las metodologías exactas más empleadas son: I) modelos de programación entera mixta (Saadouli et al., 2015) II) modelos de programación multiobjetivo (Zhang et al., 2019), III) programación por restricciones (Younespour et al., 2019; Gür et al., 2019), IV) optimización estocástica (Bovim et al., 2020), V) generación de columnas (Akbarzadeh et al., 2019) y VI) Branch and bound (Ribeiro, Leoneti, y Costa, 2018).La presente investigación propone un modelo matemático basado en programación por compromisos como estrategia de solución al problema de programación de cirugías electivas. Esta técnica ha sido ampliamente aplicada en la bibliografía en distintos problemas y sectores en los casos donde se quieren atender al mismo tiempo diferentes objetivos.

En el sector de generación de energía se han considerado aplicaciones con objetivos de costos de operación, gestión de las emisiones de CO2 y complementariedad de fuentes de energía (Canales et al., 2020; Miao et al., 2020). En el sector financiero se han desarrollado aplicaciones principalmente para la selección de portafolios, (Amiri, et al.,2011; Bilbao-Terol et al., 2006). En el sector logístico se encontraron estudios enfocados en selección de rutas aéreas, (Chang et al., 2019) y planeación de producción con criterios que incluyen el impacto social, y la generación de utilidades y reducciones de costos (Allouche et al., 2009). En el sector agrícola se evidencia el uso de técnicas multiobjetivo que permitan la reducción de la demanda química de oxígeno, y la concentración de hierro en el tratamiento de aguas residuales provenientes de la producción de café soluble (Ibarra-Taquez, et al.,2011). Otro sector en los cuales se ha implementado técnicas de optimización multiobjetivo, se encuentra el sector plástico de polipropileno, donde se muestra un mejoramiento del $12,6 \%$ en los costos asociados al programa de planeación, al mismo tiempo se optimiza el Índice de degradación a la procesabilidad lo que genera una buena calidad del polipropileno reciclado (Vélez- \& López 2017). 
Entre las aplicaciones de metodologías empleadas a la programación por compromisos se encontraron enfoques de toma de decisión difusos (Khodaei et al., 2018; Miao et al., 2020), técnicas de correlación de coeficientes (Canales et al., 2020), distancia de la función objetivo en valores nadir (Amiri et al., 2011) y programación de compromiso con restricciones de azar (Abdelaziz et al., 2007). Igualmente, dentro de la literatura consultada se evidencian funciones de desempeño como: maximizar el mayor número cirugías preferenciales programadas (Guido y Conforti, 2017);minimización de los tiempos ociosos (Ali, Lamsali, y Othman, 2019) y (Erekat et al, 2019) este objetivo busca asignar el menor número posible de médicos tal que la demanda de cirugías obligue al uso eficiente de los mismo reduciendo los tiempos ociosos entre cirugías consecutivas, lo cual garantiza el bienestar entre los médicos de tal modo que los días que estos son programados tienen el mayor número de cirugías según su capacidad quedando libre los otros días, por otro lado se encuentra la maximización del número de clientes atendidos, esta medida de desempeño se enfoca en la satisfacción de pacientes y el uso de los quirófanos, para ello se busca maximizar la cantidad de cirugías programadas en el horizonte de tiempo planeado, lo cual indirectamente mejora la utilización de los recursos disponibles (quirófanos y médicos) (Zhang et al., 2019).

Los sistemas de gestión de calidad definen a los clientes internos como aquellos que participan en la realización de un producto o servicio; y el cliente externo es el usuario final de dicho esfuerzo. En sistemas de atención hospitalarias se han definido como clientes internos a médicos y enfermeras (Milina-garduza y Rivera-barragán, 2012) y a los clientes externos como los pacientes demandantes de estos servicios. En el contexto de la programación de cirugías electivas podemos identificar diferentes actores que se ven afectados por estas decisiones; por una parte, se encuentran los pacientes o clientes externos que buscan su bienestar al ser atendidos; el personal médico cuyo esfuerzo radica en la atención de la mayor cantidad de usuarios en las condiciones de tiempo más favorables para ellos y el cuerpo administrativo que se enfoca en el uso eficiente de los recursos disponibles. La satisfacción de los distintos intereses de estos actores refleja la naturaleza multiobjetivo de este problema en una situación real de la logística hospitalaria. Es importante resaltar que satisfacción de los intereses del personal administrativo y de los médicos apunta al mejoramiento de clima laboral de estos y según lo expuesto en (del Lapo-Maza \& Bustamante-Ubilla, 2018), mejora el comportamiento prosocial en instituciones de salud, aspecto clave para garantizar un mejor servicio a los usuarios.

Como aspectos relevantes y de novedad en la presente investigación se desarrolla un modelo matemático multi objetivo para resolver el problema de programación de cirugías, que considera el cliente interno y externo a partir de la inclusión de sus referencias como funciones de desempeño normalizadas en un proceso de optimización por compromisos. Además, se involucra una adaptación novedosa de una medida de preferencia centrada en la holgura y el tiempo de duración de la cirugía, basada en las heurísticas del problema del programación.

\section{METODOLOGÍA}

La metodología se presenta en varias subsecciones: programación por compromisos, supuestos del modelo, funciones objetivo, maximización de la preferencia total, minimización de los tiempos ociosos, maximización del número de clientes .formulación de la función objetivo del problema por compromisos, y finalmente las restricciones

\section{Programación por compromisos}

La programación por compromisos hace parte de los métodos de optimización multiobjetivo, de manera general en estos se busca obtener un conjunto de soluciones eficientes para todas las funciones objetivos en el problema, este conjunto de soluciones es llamado frontera de Pareto o conjunto de soluciones no dominadas (Aranda y Orjuela, 2015). Una vez generado el conjunto de soluciones de la frontera de Pareto, los tomadores de decisión deben enfrentarse al problema de definir qué solución es más conveniente para su implementación. En la programación por compromisos se consideran los múltiples objetivos en un solo modelo y se busca encontrar una única solución que represente el balance o la eficiencia de estos objetivos (Zelany ,1974). En vista de que en términos prácticos los encargados de realizar la programación de cirugías deben implementar una única solución, y en aras de la practicidad del proceso, en este trabajo empleamos la programación por compromisos ya que como se expresó considera múltiples objetivos y arroja una única solución, resolviendo el problema en una sola fase, sin tener que pasar por el proceso adicional de selección de puntos dentro de una frontera de Pareto.

La programación por compromisos tiene los siguientes elementos, el vector de valores ideales o alternativa para los $\mathrm{n}$ objetivos del problema a considerar representando en la ecuación 1, la distancia normalizada adimensional representado en la ecuación 2; se puede entonces expresar el problema de programación por compromisos como la minimización de la distancia entre la satisfacción de los objetivos y sus valores ideales, donde el conjunto de variables $X$ este sujeto a un conjunto de restricciones lineales $F$ (ecuaciones 3 y 4 ). 


$$
\begin{aligned}
& f^{*}=\left(f_{1}^{*}, \ldots f_{i}^{*}, \ldots f_{n}^{*}\right) \\
& d_{i}=\frac{\left[f_{i}^{*}-f_{i}(x)\right]}{\left[f_{i}^{*}-f_{\mathrm{t}_{i}}\right]} \\
& \operatorname{Min}_{L}=\sum_{i}^{n} d_{i} \\
& X \in F
\end{aligned}
$$

Además, cabe resaltar la importancia del uso de técnicas de elección de soluciones bajo enfoques multicriterio como lo es la programación por compromiso, la cual permite escoger una solución eficiente del conjunto de soluciones dominantes arrojadas, logrando el máximo beneficio en las medidas de desempeño asociadas al modelo. Con el propósito de resolver el problema de programación de cirugías en el que se consideren los intereses de los clientes externos representados por los pacientes y los clientes internos representados por el personal médico y administrativo se plantea un modelo matemático multiobjetivo resuelto a partir de programación por compromiso buscando la maximización de la eficiencia de los objetivos, a continuación, se presentan cada uno de los componentes de la formulación del modelo.

\section{Supuestos del modelo}

A continuación, se presentan los supuestos propuestos en el desarrollo de modelo matemático establecido: I) Según complejidad de la enfermedad del paciente, se tiene una fecha máxima en la que la cirugía podría realizarse sin generar un compromiso mayor y se sabe que médico la realizará. II)Los quirófanos son multifuncionales.

\section{Funciones Objetivo}

El modelo matemático desarrollado considera 3 objetivos; los cuales fueron escogidos por su predominación en la literatura: I) Maximizar la prioridad de los pacientes (FObj_MaxPrf) (Guido y Conforti, 2017); II) Minimizar la ociosidad (FObj_MinOcio) (Ali et al., 2019); III) Maximizar el número de pacientes (FObj_MaxCli) (Zhang et al., 2019).

\section{Maximización de la preferencia total.}

En la presente investigación se propone definir dicha preferencia teniendo en cuenta la urgencia de esta, su duración y la fecha máxima de asignación permitida; así pues, la preferencia de una cirugía puede ser expresada de la siguiente manera:

$$
\begin{aligned}
& \text { Preferencia }_{i}=\frac{\text { Prioridad }}{\text { Duración }}{ }^{*} e^{\frac{-(\text { FechaMaxima*6*24-Duración })}{\text { Duración Promedio }}} \\
& \text { preftotal }=\sum_{i \in I, d \in D, q \in Q} Y_{i \neq 0} Y_{\text {idq }}{ }^{*} \text { Preferencia } a_{i}
\end{aligned}
$$

Con el uso de esta medida de preferencia, se busca que las cirugías que están atrasadas tengan la mayor ponderación, para las cirugías con holgura positiva la ponderación decrece a medida que aumenta dicha holgura. Esta medida fue basada en la propuesta de (Sipper et al. 1998). La función objetivo que considera la preferencia es mostrada en (6) en esta se busca maximizar la preferencia total (preftotal).

Minimización de los tiempos ociosos.

Este objetivo ha sido adaptado de modelos propuestos por autores como (Ali, Lamsali, y Othman, 2019) y (Erekat et al, 2019).

$$
\text { num_med }=\sum_{m \in \text { Med,d } \in \text { D }} A_{m d}
$$

Maximización del número de clientes.

Esta medida ha sido acondicionada de otros autores (Zhang et al., 2019). 


$$
\text { num_clie }=\sum_{i \in I, d \in D ; q \in Q} Y_{\text {idq }}
$$

Formulación de la función objetivo del problema por compromisos

La ecuación 9 resume la formulación de la función objetivo desarrollada en el modelo matemático propuesto, teniendo en cuenta los distintos objetivos considerados anteriormente. Esta función es acorde a la ecuación 3 , la cual representa la suma de las distancias de sus objetivos a sus ideales normalizados según se presentan en la ecuación 2.

$$
\text { Max FObj_MaxPrf+FObj_MinOcio+FObj_MaxCli }
$$

\section{Restricciones}

Las ecuaciones 10,11 y 12 definen el valor de la distancia normalizada adimensional de las medidas de desempeño con respecto a su óptimo en una escala de 0 a 1, acorde a lo que se presenta en la ecuación 2. Las ecuaciones 13 y 14 asegura una sola transición entre dos cirugías programadas de un mismo médico o quirófano. Las ecuaciones 15 y 16 garantizan la continuidad entre las cirugías en quirófanos y por médico.

$$
\begin{aligned}
& \text { FObj_MaxPrf = -VAI_FObj_MaxPrf+ preftotal }{ }^{*} \text { Pen_FObj_MaxPrf } \\
& \text { FObj_MinOcio }=\text { VAI_FObj_MinOcio }- \text { num_med }{ }^{*} \text { Pen_FObj_MinOcio } \\
& \text { FObj_MaxCli }=- \text { VAI_FObj_MaxCli }+\sum_{i \in I, d \in D ; q \in Q} \frac{Y_{\text {idq }}}{\operatorname{card}(I)} \\
& \sum_{d \in D} X_{m d i j}+\sum_{d \in D} X_{m d j i} \leq 1 \quad \forall m \in \operatorname{Med}, i \in \mathbb{I M}_{\{m\}}, j \in \mathbb{I M}_{\{m\}} / i \neq j \\
& \sum_{q \in Q ; d \in D} G_{q d i j}+\sum_{q \in Q ; d \in D} G_{q d i j} \leq 1 \quad \forall i \in l, j \in l / i \neq j \\
& \sum_{i \in I M_{m} \cup 0, i<>h} X_{m d i h}=\sum_{j \in I M_{m} \cup 0, j<>h} X_{m d h j} \quad \forall m \in \text { Med,helM } M_{m}, d \in D \\
& \sum_{\substack{i \in|U 0| \\
i \neq h}} G_{q d i h}=\sum_{\substack{j \in l U 0 \mid \\
j \neq h}} G_{q d h j} \quad \forall h \in l, q \in Q, d \in D
\end{aligned}
$$

Las ecuaciones 17 y 18 relacionan la secuencia con la programación de la cirugía, garantizando que solo se pueda dar secuencia a cirugías de pacientes que han sido programadas para un día en particular. Las ecuaciones 19, 20, 21, 22, 23, 24, 25 y 26 garantizan unicidad en el inicio, finalización y programación de una cirugía en cada secuencia por médico o quirófano.

$$
\begin{aligned}
& \sum_{h \in I M M_{\{m\}} \cup 0} X_{\text {mdih }}=\sum_{q \in \mathrm{Q}} \mathrm{Y}_{\mathrm{idq}} \quad \forall m \in \operatorname{Med}, \mathrm{i} \in \mathbb{I M}_{\{m\}}, \mathrm{d} \in \mathrm{D} \\
& \sum_{\substack{\text { helU0। } \\
i \neq h}} \mathrm{G}_{\text {qdih }}=Y_{\text {idq }} \quad \forall i \in I, q \in Q, d \in D \\
& \sum_{i \in \mathbb{M}_{\{m\}}} x_{m d 0 i} \leq 1 \quad \forall m \in \operatorname{Med}, d \in D \\
& \sum_{i \in I M_{\{m\}}} X_{\text {mdio }} \leq 1 \quad \forall m \in \text { Med, } d \in D \\
& \sum_{\substack{h \in I M_{\{m\}} \cup 0 ; d \in D \mid \\
i \neq h}} X_{m d i h} \leq 1 \quad \forall m \in \text { Med, i } \in \mathbb{I M}_{\{m\}} \\
& \sum_{\substack{h \in I M_{\{m\}} \cup 0 ; ; d \in D \mid \\
i \neq h}} X_{m d h i} \leq 1 \quad \forall m \in \text { Med, i } \in \mathbb{I M}_{\{m\}}
\end{aligned}
$$




$$
\begin{array}{lc}
\sum_{i \in \mid} G_{q d 0 i} \leq 1 & \forall d \in D, q \in Q \\
\sum_{i \in \mid} G_{q d i 0} \leq 1 & \forall d \in D, q \in Q \\
\sum_{\substack{d \in d ; q \in Q ; h \in \| \mid \\
i \neq h}} G_{q d i h} \leq 1 & \forall i \in I \\
\sum_{d \in d, q \in Q, h \in l} G_{l i \neq h} & \forall i \in I
\end{array}
$$

Las ecuaciones $27,28,29,30,31,32,33$, y 34 regulan las relaciones de precedencias en la programación asociadas por un lado a los médicos y las diferentes cirugías que estos realizan en todos los quirófanos a lo largo de un día y por otro lado a los quirófanos y todas las cirugías que se programan en estos, garantizando de este modo la sincronización entre la planificación del médico y la del quirófano.

$$
\begin{aligned}
& \mathrm{e} 1_{\mathrm{i}}+\text { delta }_{\mathrm{mdij}} \leq \mathrm{s} 1_{\mathrm{j}}+\left(1-\mathrm{X}_{\mathrm{mdij}}\right)^{*} \mathrm{~T} \quad \forall \mathrm{m} \in \mathrm{Med}, \mathrm{i} \in \mathrm{IM}_{\{\mathrm{m}\}}, \mathrm{j} \in \mathrm{IM}_{\{\mathrm{m}\}}, \mathrm{d} \in \mathrm{D} \mid \mathrm{i} \neq \mathrm{j} \\
& \mathrm{e} 1_{\mathrm{i}}+\text { delta }_{\mathrm{mdij}_{\mathrm{ij}}} \geq \mathrm{s} 1_{\mathrm{j}}-\left(1-\mathrm{X}_{\mathrm{mdij}}\right)^{\star} \mathrm{T} \quad \forall \mathrm{m} \in \mathrm{Med}, \mathrm{i} \in \mathrm{IM}_{\{\mathrm{m}\}}, \mathrm{j} \in \mathrm{IM}_{\{\mathrm{m}\}}, \mathrm{d} \in \mathrm{D} \mid \mathrm{i} \neq \mathrm{j} \\
& \mathrm{e} 1_{\mathrm{i}} \geq \mathrm{s} 1_{\mathrm{i}}+\sum_{\mathrm{d} \in \mathrm{D}, \mathrm{q} \in \mathrm{Q}} \mathrm{Y}_{\mathrm{idq}}{ }^{*} \mathrm{dur}_{\mathrm{i}} \quad \forall \mathrm{m} \in \mathrm{Med}, \mathrm{i} \in \mathrm{IM}_{\{\mathrm{m}\}} \\
& \mathrm{e} 1_{\mathrm{i}} \leq \mathrm{s} 1_{i}+\sum_{\mathrm{d} \in \mathrm{d}, \mathrm{q} \in \mathrm{Q}} \mathrm{Y}_{\mathrm{idq}}{ }^{*} \mathrm{dur} \mathrm{r}_{\mathrm{i}} \quad \forall \mathrm{i} \in \mathrm{I} \\
& \mathrm{s} 1_{\mathrm{i}} \leq \sum_{\mathrm{d} \in \mathrm{D}, \mathrm{q} \in \mathrm{Q}} \mathrm{Y}_{\mathrm{idq}}{ }^{*}\left(\mathrm{~T}-\mathrm{dur}_{\mathrm{i}}\right) \quad \forall \mathrm{m} \in \operatorname{Med}, \mathrm{i} \in \mathrm{IM}_{\{\mathrm{m}\}} \\
& \mathrm{e} 1_{\mathrm{i}} \leq \sum_{\mathrm{d} \in \mathrm{D}, \mathrm{q} \in \mathrm{Q}} \mathrm{Y}_{\mathrm{idq}}{ }^{*} \mathrm{~T} \quad \forall \mathrm{m} \in \operatorname{Med}, \mathrm{i} \in \mathrm{IM}_{\{\mathrm{m}\}} \\
& s 1_{i} \leq\left(1-G_{q d 0 i}\right)^{*} T \quad \forall i \in I, q \in Q, d \in D \\
& \mathrm{e} 1_{\mathrm{i}} \leq \mathrm{s} 1_{\mathrm{j}}+\left(1-\mathrm{G}_{\mathrm{qdij}}\right) * T \quad \forall \mathrm{q} \in \mathrm{Q}, \mathrm{i} \in \mathrm{I}, \mathrm{j} \in \mathrm{I}, \mathrm{d} \in \mathrm{D} \mid \mathrm{i} \neq \mathrm{j}
\end{aligned}
$$

Las ecuaciones 35 y 36 obligan que las cirugías de un mismo médico que se programan consecutivas se realicen el mismo día. Las ecuaciones 37 y 38 determina que dos cirugías programadas, de ser consecutivas entre ellas deben ejecutarse el mismo día y en la misma sala. Las ecuaciones 39 y 40 aseguran la secuencia y asignación de cirugías consecutivas.

$$
\begin{aligned}
& \sum_{\mathrm{q} \in \mathrm{Q}} \mathrm{Y}_{\mathrm{idq}} \leq \sum_{\mathrm{q} \in \mathrm{Q}} \mathrm{Y}_{\mathrm{jdq}}+\left(1-\mathrm{X}_{\mathrm{mdji}}-\mathrm{X}_{\mathrm{mdij}}\right) \quad \forall \mathrm{m} \in \operatorname{Med}, \mathrm{i} \in \mathrm{IM}_{\{\mathrm{m}\}}, \mathrm{j} \in \mathrm{IM}_{\{\mathrm{m}\}}, \mathrm{d} \in \mathrm{D} \mid \mathrm{i} \neq \mathrm{j} \\
& \sum_{\mathrm{q} \in \mathrm{Q}} \mathrm{Y}_{\mathrm{idq}} \geq \sum_{\mathrm{q} \in \mathrm{Q}} \mathrm{Y}_{\mathrm{jdq}}-\left(1-\mathrm{X}_{\mathrm{mdji}}-\mathrm{X}_{\mathrm{mdij}}\right) \quad \forall m \in \operatorname{Med}, \mathrm{i} \in \mathrm{IM}_{\{\mathrm{m}\}}, \mathrm{j} \in \mathrm{IM}_{\{\mathrm{m}\}}, \mathrm{d} \in \mathrm{D} \mid \mathrm{i} \neq \mathrm{j} \\
& Y_{\text {idq }} \leq Y_{\text {jdq }}+\left(1-G_{q d j i}-G_{q d i j}\right) \quad \forall i \in I, j \in I, d \in D, q \in Q \mid i \neq j \\
& Y_{\text {idq }} \geq Y_{j d q}-\left(1-G_{q d j i}-G_{q d i j}\right) \quad \forall i \in I, j \in I, d \in D, q \in Q \mid i \neq j \\
& \mathrm{G}_{\mathrm{qdij}} \leq \mathrm{X}_{\mathrm{mdij}}+\left(2-\mathrm{Y}_{\mathrm{idq}}-Y_{\mathrm{jdq}}\right) \quad \forall m \in \operatorname{Med}, \mathrm{i} \in \mathrm{IM}_{\{\mathrm{m}\}}, \mathrm{j} \in \mathrm{IM}_{\{\mathrm{m}\}}, \mathrm{d} \in \mathrm{D}, \mathrm{q} \in \mathrm{Q} \mid i \neq j \\
& \mathrm{G}_{q d i j} \geq X_{m d i j}-\left(2-Y_{i d q}-Y_{j d q}\right) \quad \forall m \in M e d, i \in I_{\{m\}}, j \in I_{\{m\}}, d \in D, q \in Q \mid i \neq j
\end{aligned}
$$

La ecuación 41 obliga que las cirugías deben programarse a lo sumo una vez. La ecuación 42 controla la capacidad disponible en los quirófanos, mientras que la ecuación 43 controla la capacidad del médico. Finalmente, las ecuaciones 44, 45, 46, 47 permiten establecer el número de días que son programados los médicos. 


\begin{tabular}{|c|c|c|}
\hline$\sum_{d \in d, q \in Q} Y_{i d q} \leq 1$ & $\forall \mathrm{i} \in \mathrm{l} / \mathrm{i} \neq 0$ & (41) \\
\hline$\sum_{i \in \mid} Y_{i d q}{ }^{*} d u r_{i} \leq C a p$ & $\forall d \in D, q \in Q$ & (42) \\
\hline$\sum_{i \in I M_{\{m\}}, q \in Q} Y_{i d q}^{*}{ }^{*} \operatorname{dur}_{i} \leq C_{a p}{ }^{*} M_{m d}$ & $\forall \mathrm{d} \in \mathrm{D}, \mathrm{m} \in \mathrm{Med}$ & (43) \\
\hline$\sum_{i \in I M_{\{m\}}, q \in Q} Y_{i d q} \leq A_{m d}{ }^{*} \operatorname{Card}\left(I_{\{m\}}\right)$ & $\forall \mathrm{m} \in$ Med, $\mathrm{d} \in \mathrm{D}$ & (44) \\
\hline$\sum_{i \in \mid \mathbb{M}_{\{m\}}, q \in Q} Y_{i d q} \geq A_{m d}$ & $\forall \mathrm{m} \in$ Med, $\mathrm{d} \in \mathrm{D}$ & (45) \\
\hline$\sum_{d \in D} A_{m d} \geq 1$ & $\forall \mathrm{m} \in \mathrm{Med}$ & (46) \\
\hline$\sum_{d \in D} A_{m d} \leq 5$ & $\forall m \in$ Med & (47) \\
\hline
\end{tabular}

\section{RESULTADOS Y DISCUSIÓN}

El modelo propuesto fue validado en una clínica del centro de Valle del Cauca, Colombia, la cual atiende servicios de salud altamente especializados en cerca de una docena de municipios del centro y norte del departamento. El modelo de optimización fue implementado en AMPL y el MILP fue solucionado con GUROBI 9.0.2, buscando satisfacer las necesidades de 77 pacientes (p), tiempos de cirugía entre 30 y 210 minutos, contando con 26 médicos disponibles, en un horizonte de planeación de 5 días, 10 periodos de 30 minutos por día para programar asignaciones y 4 Quirófanos. En este caso concreto los tiempos de duración de las cirugías se basan en históricos, para ello se tomaron los promedios y se sumaron 2 desviaciones estándar por arriba de la media en tiempo, de tal modo, que asumiendo una distribución normal, se pueda garantizar que el $\mathbf{9 7 . 7 \%}$ de las veces las cirugías se van a poder realizar en el tiempo planeado.

A diferencia de lo presentado en (Londoño, Orejuela y Gil, 2019), donde solamente se asigna las cirugías a los quirófanos y la secuenciación se realiza en un paso posterior al modelo de optimización empleando una regla de despacho; un enfoque subóptimo; en la presente investigación, la asignación y la secuenciación de médicos y quirófanos son decisiones del modelo de optimización generando complejidad, pero ganando generalidad y eliminando esta suboptimalidad. Adicionalmente la consideración de la secuenciación también permite la definición de nuevas medidas de desempeño asociadas al mismo.

Se han desarrollado 5 casos para la ejecución del modelo; 4 de estos casos propuestos se enfocan en la eficiencia de las funciones objetivos, cada uno de estos presenta una variación en su función objetivo modificando la prioridad de las distintas funciones; el último caso propuesto se basa en el balance de estas. Se plantean 6 medidas de desempeño para evaluar los casos desarrollados, las tres primeras medidas (Cercanía Óptimo FObj_MaxPrf (Guido y Conforti, 2017), Cercanía Óptimo FObj_MinOcio (Ali, Lamsali, y Othman, 2019), Cercanía Óptimo FObj_Max (Zhang et al., 2019)) miden la cercanía porcentual entre las distintas funciones objetivo y su valor ideal, la cuarta medida (Índice de preferencia total) mide el nivel de cirugías prioritarias programadas, la quinta medida (Promedio médicos programados por día) mide la utilización del personal médico y está relacionada con la función objetivo FObj_MinOcio, por último la sexta medida de desempeño mide el número total de pacientes atendidos en el periodo de planeación y se relacionada con la función objetivo FObj_MaxCli .

En el caso 1 se le da prioridad a la cantidad de pacientes atendidos (FObj_MaxCli), se puede observar cómo los quirófanos mantienen un alto nivel de ocupación y presenta el mayor número promedio de médicos asignados de todos los casos; a su vez se observa un valor alto en su cercanía con el óptimo FObj_MaxPrf, lo cual se traduce en un gran número de cirugías prioritarias asignadas. La figura 1 muestra los resultados de programación en este caso. El caso 2 da prioridad a la medida de desempeño de Cercanía Óptimo FObj_MaxPrf, enfocada a la asignación de cirugías preferenciales, esto lleva a una de las más altas asignaciones de pacientes y utilización de recursos disponibles de los casos presentados (médicos y quirófanos). La figura 2 muestra los resultados de programación en este caso. 


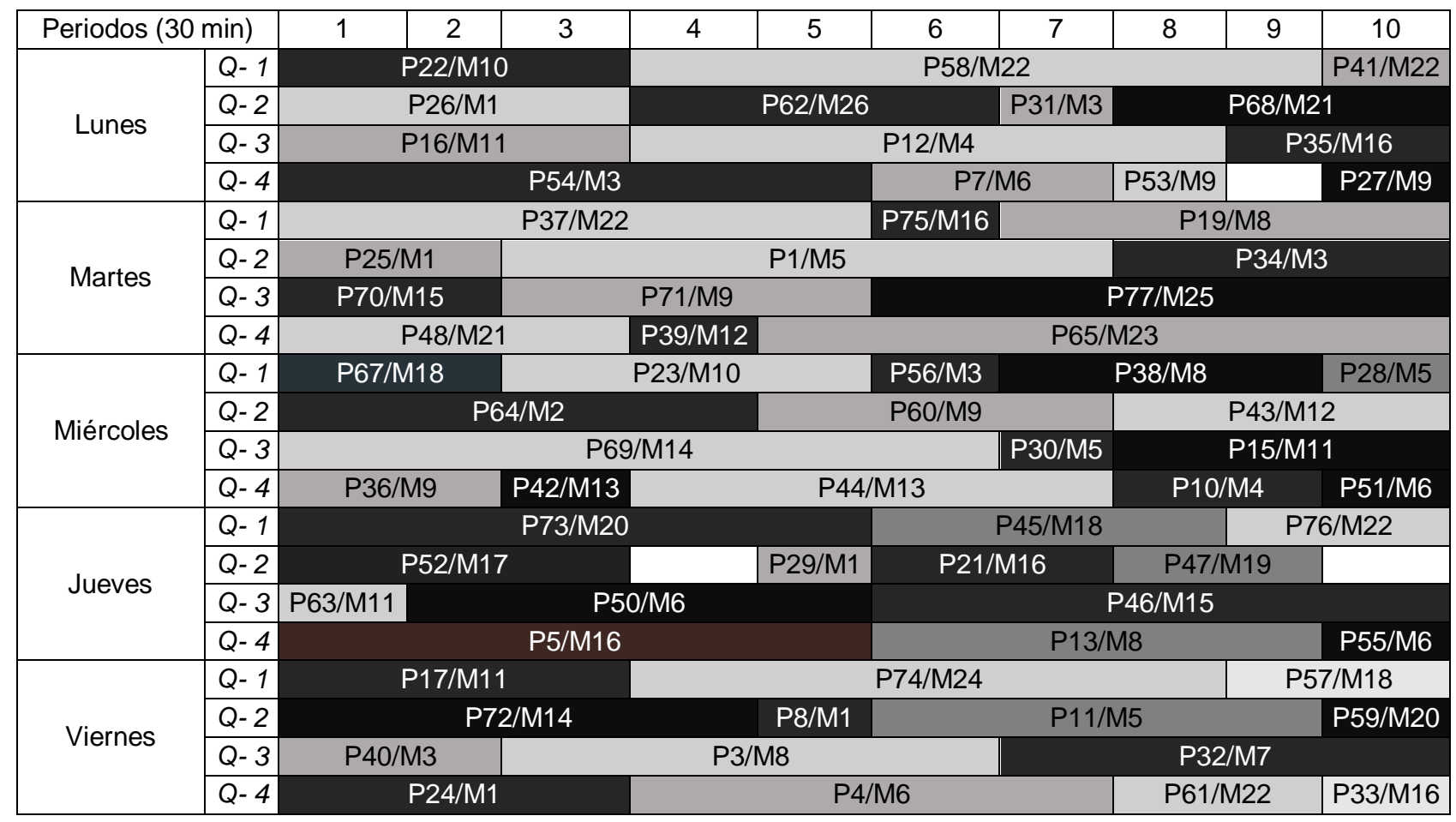

Fig. 1. Programación propuesta para el caso 1: Prioridad a número de clientes atendidos

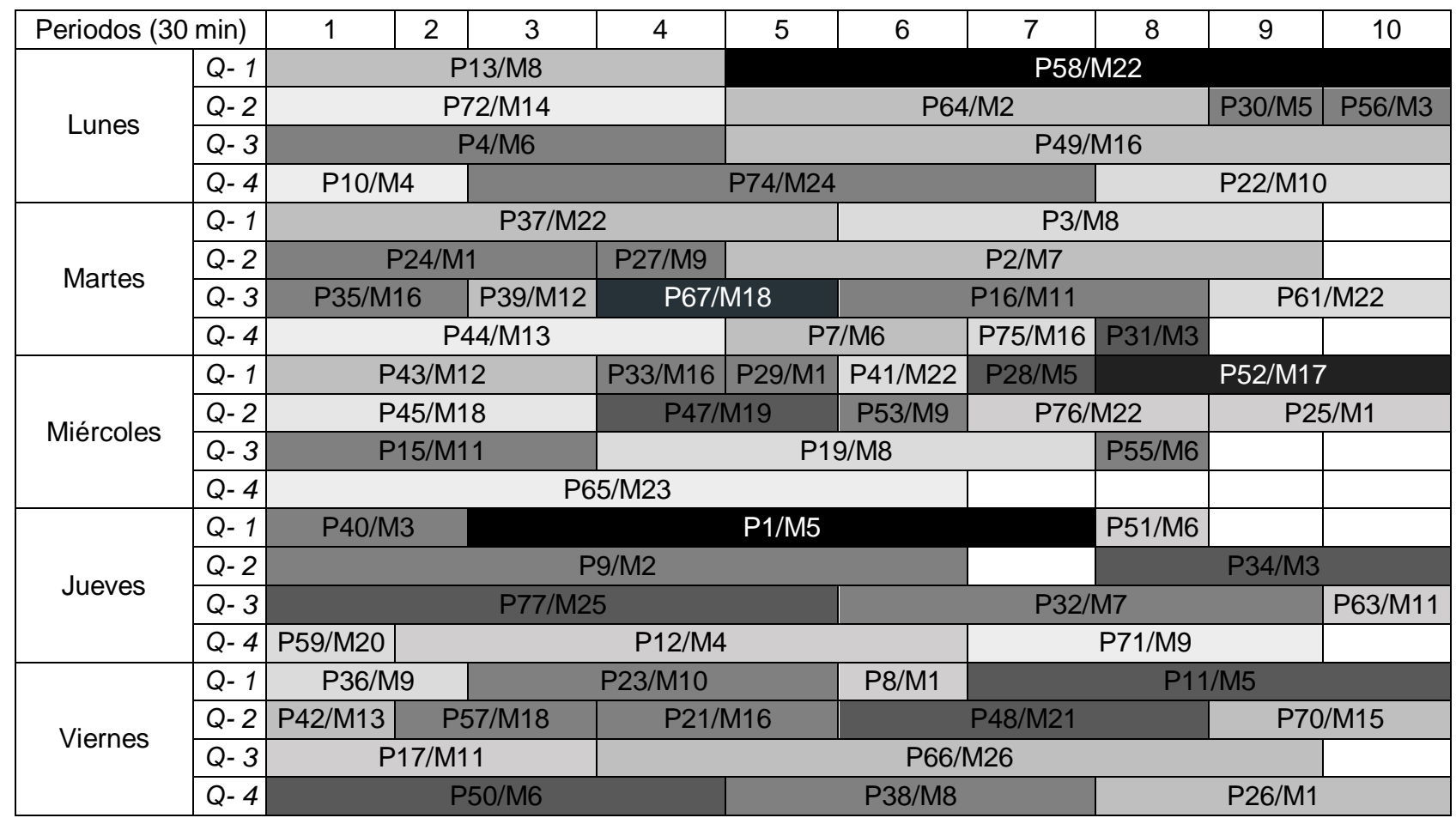

Fig. 2. Programación propuesta para el caso 2. Prioridad a la urgencia y la duración

El caso 3 da prioridad a la utilización del personal médico disponible o reducir su ociosidad (FObj_MinOcio) lo cual se aprecia en el menor número promedio de médicos asignados por día; sin embargo, dado que cada médico debe ser asignado al menos un día a la semana, pero no hay un mínimo de cirugías a asignar, el modelo busca programar la menor cantidad de médicos posible, lo cual resulta en un menor número de pacientes asignados y utilización de quirófanos. La figura 3 muestra los resultados de programación con en este caso. El caso 4 ejecuta el modelo multi objetivo planteado en la ecuación 9 enfocada en la eficiencia de sus métricas de desempeño, para ello se toma los resultados de los casos 1,2 y 3 para extraer los valores óptimos y anti ideales (peores resultados) de las funciones objetivo necesarios para la ejecución de este caso; este caso demuestra buenos resultados en todas las medidas de desempeño propuesta. La figura 4 muestra los resultados de programación con en este caso. 


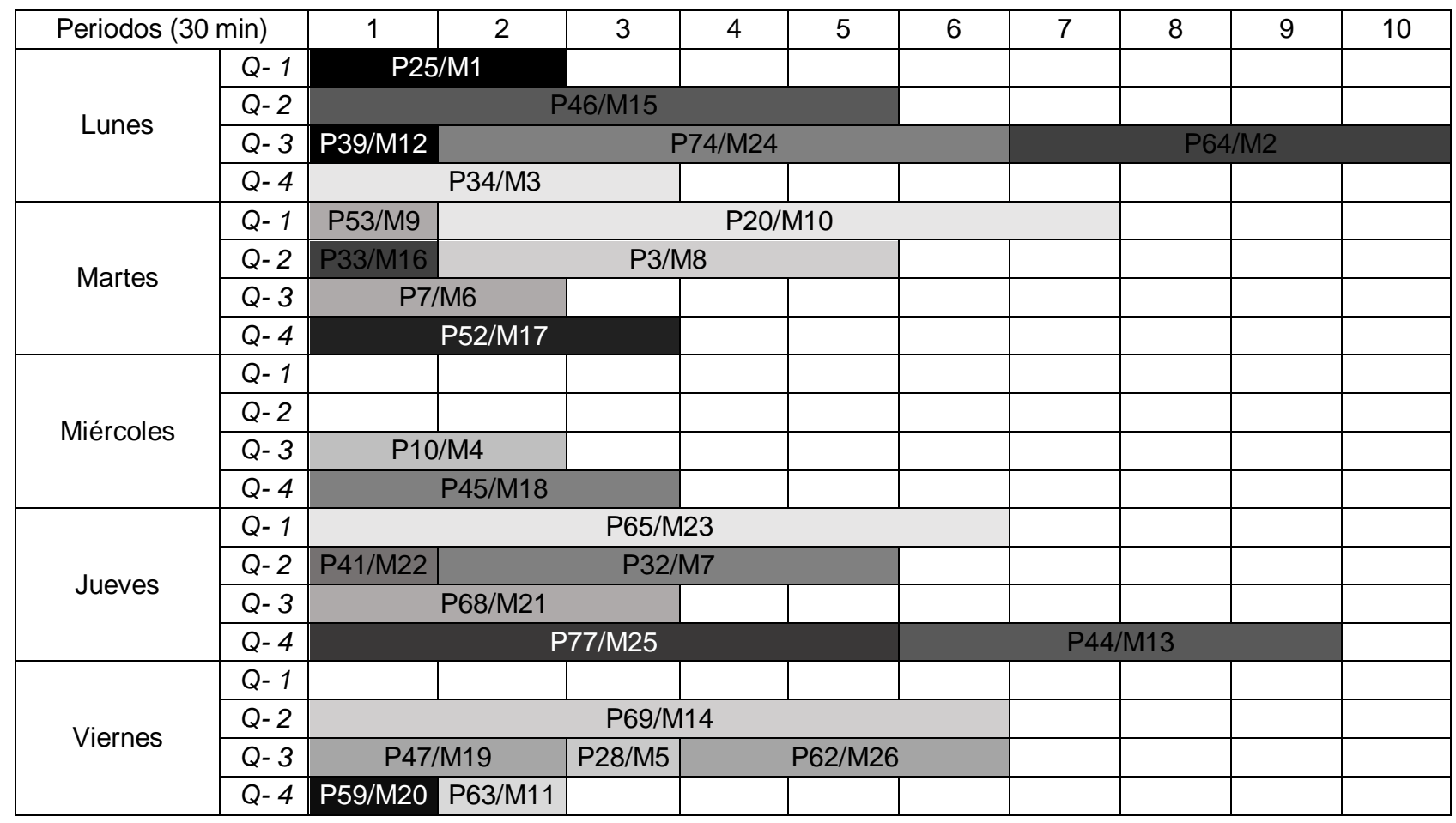

Fig. 3. Programación propuesta para el caso 3: Prioridad a reducir el tiempo ocioso de los médicos

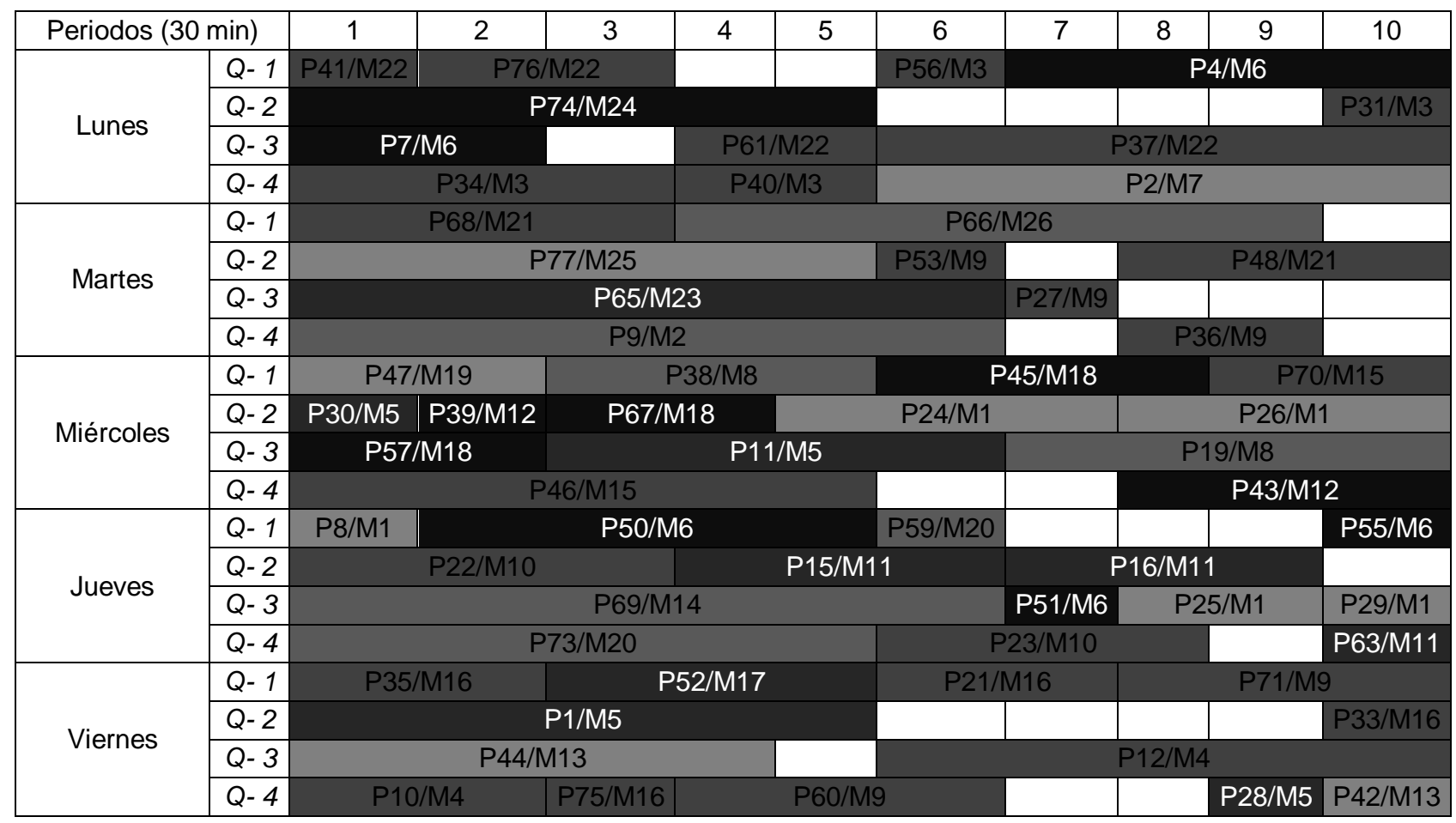

Fig. 4. Programación propuesta para el caso 4: Eficiencia en las métricas normalizadas

El caso 5 ejecuta el modelo enfocado en el balance de sus funciones objetivos, se presentan resultados idénticos al caso 4 en sus medidas de desempeño excepto en la de Cercanía Óptimo FObj_MaxPrf, la cual evidencia una leve disminución, es importante resaltar que el balance de los objetivos busca que el valor del objetivo más pequeño sea lo más alto posible por esto el número de pacientes atendidos es el objetivo más bajo, y al no poder mejorarse esta medida sin empeorar las otras por debajo de este límite las otras funciones de desempeño no son obligados a obtener un mejor valor de tal modo que se logran mejores resultados cuando se mejora la eficiencia en contraste con el balance. La figura 5 muestra los resultados de programación con en este caso. 


\section{Formulación de la función objetivo-alternativa}

Buscando que el desempeño de las funciones objetivos propuestas sean lo más cercanas posibles, se propone reemplazar la ecuación 9 por la ecuación 48, de tal modo que se maximice la distancia al objetivo ideal más baja entre los tres objetivos. A su vez se define que la variable de balance OBC se limite como la función objetivo con peor desempeño, esto se representa en las ecuaciones 49,50 y 51 . La tabla 1 resume el desempeño de los casos propuestos para las medidas de desempeño desarrolladas

$\operatorname{Max} \mathrm{OBC}$

Restricciones

FObj_MaxPrf $\leq O B C$

FObj_MinOcio $\leq O B C$

FObj_MaxCli $\leq O B C$

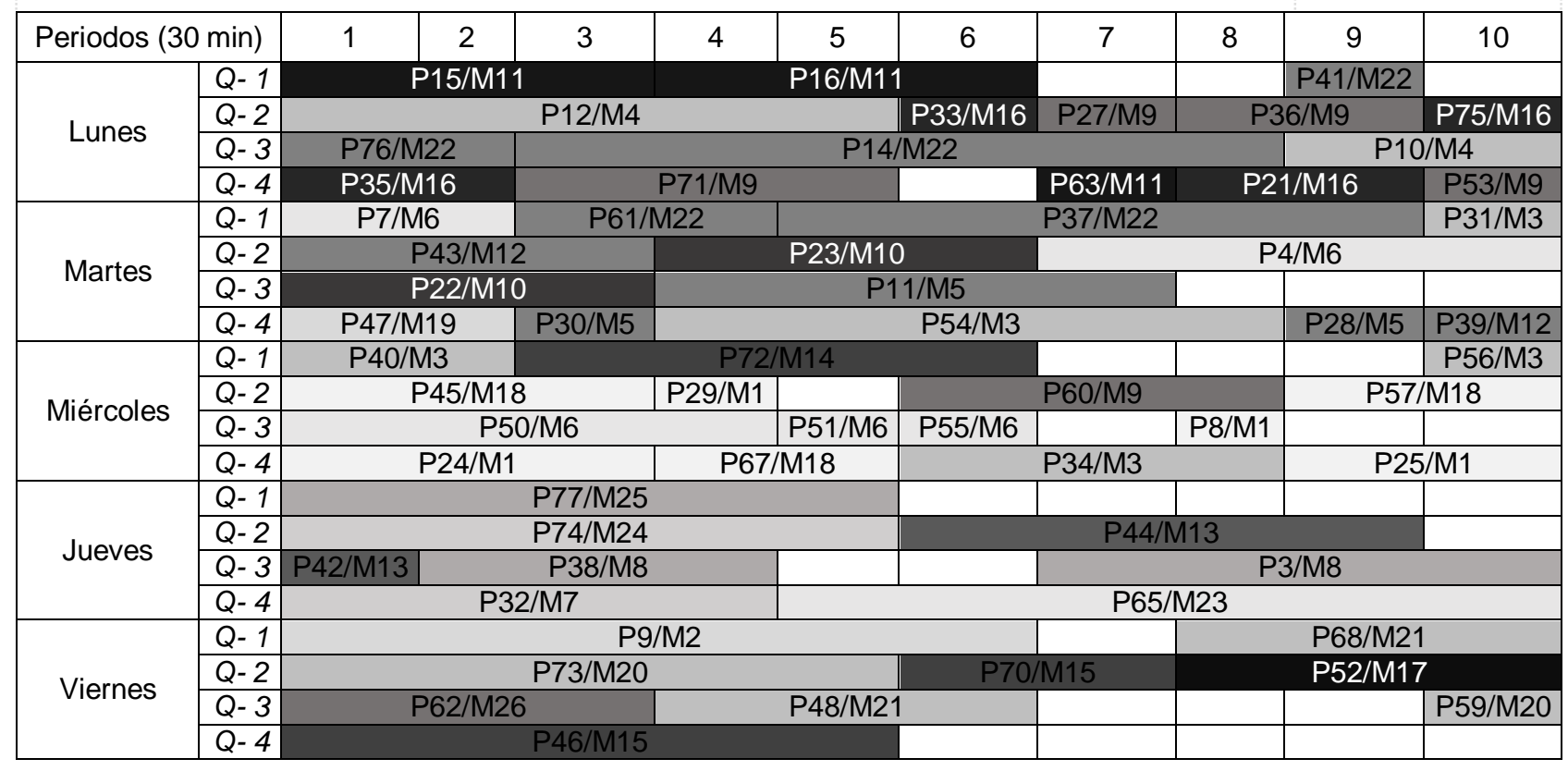

Fig. 5. Programación propuesta para el caso 5: balance entre objetivos

Tabla 1: Desempeño instancias

\begin{tabular}{|c|c|c|c|c|c|c|}
\hline Casos & $\begin{array}{c}\text { Cercanía } \\
\text { Óptimo } \\
\text { FObj_MaxPrf }\end{array}$ & $\begin{array}{c}\text { Cercanía } \\
\text { Óptimo } \\
\text { FObj_MinOcio }\end{array}$ & $\begin{array}{c}\text { Cercanía } \\
\text { Óptimo } \\
\text { FObj_MaxCli }\end{array}$ & $\begin{array}{c}\text { Índice de } \\
\text { preferencia total }\end{array}$ & $\begin{array}{c}\text { Promedio } \\
\text { médicos } \\
\text { programados por } \\
\text { día }\end{array}$ & $\begin{array}{c}\text { Pacientes } \\
\text { Atendidos }\end{array}$ \\
\hline 1 & $98.50 \%$ & $20.90 \%$ & $100.00 \%$ & 3.18 & 12 & 69 \\
\hline 2 & $100.00 \%$ & $23.30 \%$ & $94.20 \%$ & 3.22 & 11.8 & 65 \\
\hline 3 & $57.72 \%$ & $100.00 \%$ & $37.70 \%$ & 2.02 & 6.2 & 6 \\
\hline 4 & $97.80 \%$ & $90.70 \%$ & $89.90 \%$ & 3.18 & 6 & 62 \\
\hline 5 & $93.6 \%$ & $90.70 \%$ & $89.90 \%$ & 3.04 & 6 & 62 \\
\hline
\end{tabular}

La figura 6 muestra el desempeño comparativo de todos los casos realizados para las 6 medidas de desempeño propuestas, esto demuestra que el caso 4 presenta los mejores resultados en cuanto a eficiencia y logra que la medida de desempeño más baja sea igual a la que se obtiene cuando se busca el balance, en tal sentido maximiza la suma de las funciones objetivo y maximiza el desempeño de la función objetivo más baja, y por ello se plantea como la solución a implementar. 


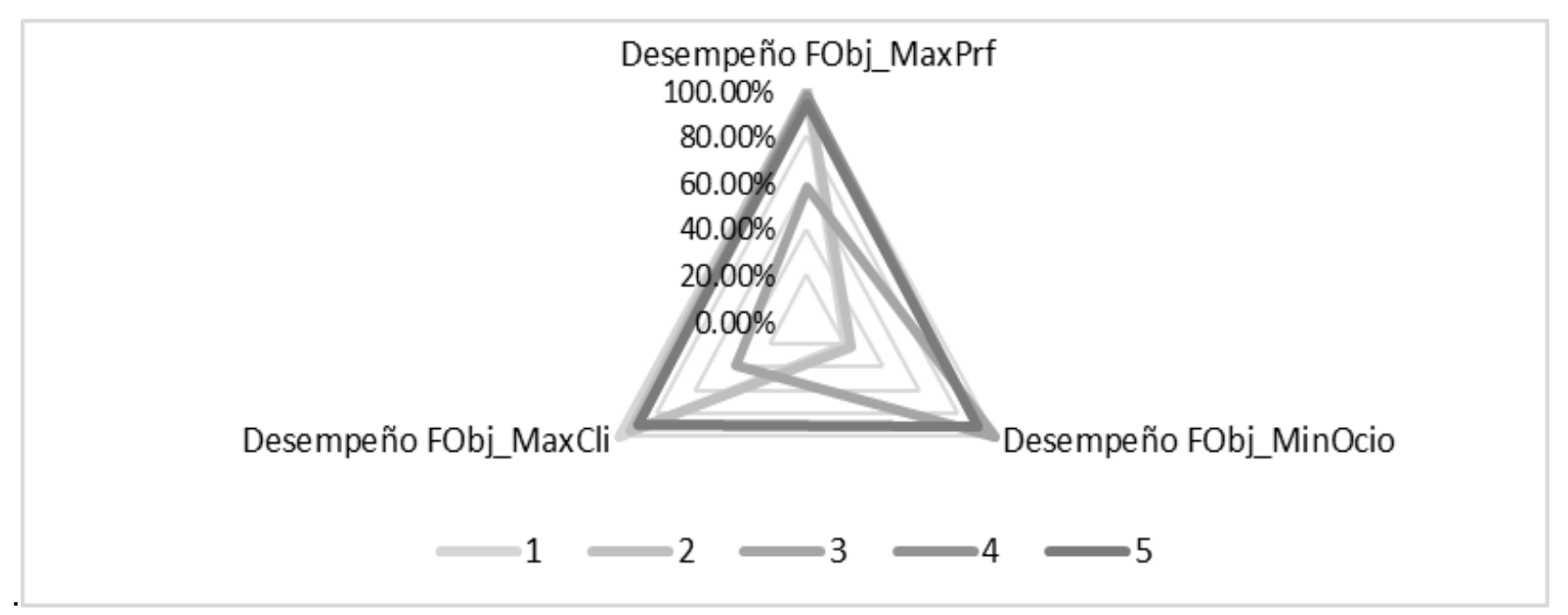

Fig. 6: Desempeño casos

Este trabajo se diferencia en la literatura gracias a la propuesta de prorratear dos características relevantes de los problemas de programación: los trabajos más pequeños vayan de primero (shortest process time) simultáneamente con los que tienen fecha de entrega más cortos vayan de primero (early due date). Esto busca garantizar que se ejecuten un mayor número de trabajos, pero también al mismo tiempo tiene en cuenta la holgura en relación con su tiempo de proceso para asignar prioridad así balanceando ambos criterios que han sido empleados en problemas tradicionales de programación pero hasta nuestro conocimiento es la primera vez que se aborda en el problema abordado, lo cual consideramos un aporte importante.

Un enfoque futuro es abordar el problema desde las técnicas centradas en fronteras de Pareto, de tal modo que se brinden diferentes soluciones eficientes al tomador de decisiones. Estos modelos son de crecimiento exponencial en el tiempo de cómputo, si incrementamos el número de médicos o el número de quirófanos, el problema puede no ser resuelto de manera óptima obligando al uso de heurísticas o técnicas de optimización avanzada, esto es una limitación que se asocia a la complejidad. Por otro lado, también se propone considerar de manera explícita la variabilidad de los tiempos de cirugía y abordar esto desde lo metodológico con optimización estocástica.

\section{CONCLUSIONES}

Los resultados presentados en este trabajo, su análisis y discusión permiten obtener las siguientes conclusiones principales:

1) Al comparar los casos 1 y 4, se observa como presentan similares niveles de rendimiento en el objetivo FObj_MaxPrf, con una diferencia de $0.5 \%$, una disminución de 7 pacientes asignados (cercana al 10\%) y la diferencia del número promedio de médicos asignados por días es el doble. Lo cual apunta a una relación inversa entre la satisfacción de pacientes y bienestar/ociosidad de los médicos.

2) Los casos 1 y 2 muestran una relación entre la maximización de cirugías prioritarias y la maximización del número de pacientes, sin embargo, la diferencia en los mismos es un indicativo de que priorizar el mayor número de cirugías no necesariamente aumenta la satisfacción de los pacientes al ignorar la urgencia del procedimiento.

3) Al analizar los casos 2 y 4, se observa una diferencia del 2\% del objetivo FObj_MaxPrf y una brecha de 3 pacientes en el número de asignaciones, sin embargo, el número promedio de médicos asignados por día pasa de 11.8 en el caso a 6 en el caso 4; sugiriendo como la asignación de un mayor número de cirugías preferenciales obligan a un mayor uso de los médicos disponibles.

4) Los casos 3 y 4 muestran un nivel similar de número promedio de médicos asignados por día, sin embargo, se encuentran discrepancias entre el número de pacientes asignados (26 en el caso 3 comparado con 69 en el caso 4) y una disparidad en el objetivo FObj_MaxPrf cercana al 40\%. Esto lleva a concluir que niveles bajos de ociosidad de médico impactan en la satisfacción de pacientes y asignación de cirugías preferenciales.

5) Al comparar los casos 4 y 5 enfocados en la eficiencia y balance de las funciones objetivos propuestas respectivamente, se ve que su desempeño es similar excepto en una leve diferencia en la función objetivo FObj_MaxPrf, lo cual sugiere que buscar el mejor desempeño para todas las funciones objetivos indirectamente reduce la brecha en el rendimiento entre ellos en este caso.

6) Al analizar el caso 3 se encuentra que la disminución del número de asignaciones de personal médico no necesariamente conduce a la reducción de la ociosidad o uso eficiente de los recursos, esto sugiere que se 
deben estudiar otras medidas de desempeño que relacionen la cantidad de médicos asignados con el número de pacientes programados.

7) Los resultados demuestran como el uso de herramientas multiobjetivo para problemas de programación de cirugías, producen buenos resultados en todas las medidas de desempeño consideradas.

8) Igualmente cabe resaltar la importancia del uso de técnicas de elección de soluciones bajo enfoques multicriterio como lo es la programación por compromiso, la cual permitió escoger una solución eficiente del conjunto de soluciones al modelo propuesto.

\section{NOTACIÓN}

\section{Conjuntos}

I,i $\in\{1 \ldots|| \mid\}$ Conjunto de pacientes a programar, indexado por el subíndice i.

Med, $m \in\{1 \ldots|M|\}$ Conjunto de médicos indexado por el subíndice $m$.

$\mathrm{D}, \mathrm{d} \in\{1 \ldots|\mathrm{D}|\}$ Conjunto de días disponibles indexado en el subíndice $d$.

$Q, q \in\{1 \ldots|Q|\}$ Conjunto de quirófanos disponibles indexado por el subíndice $q$.

IM $\epsilon\{1 \ldots|| \mid\}$ Conjunto de pacientes a programar, indexado por el subíndice i.

\section{Parámetros de decisión}

$\mathrm{PM}_{\mathrm{md}}$ : Porcentaje de disponibilidad en términos del tiempo asociado al médico $\mathrm{m}$ en el día $\mathrm{d}$.

dur flli $_{i}$ Duración de la cirugía y último día (d) en el que realiza la cirugía del paciente i.

dur $r_{i}$,lli: Duración de la cirugía y último día (d) en el que realiza la cirugía del paciente i.

VAI_FObj_MaxPrf, VAI_FObj_MinOcio, VAI_FObj_MaxCli: Valor anti ideal (peor posible) para los objetivos FObj_MaxPrf, FObj_MinOcio y FObj_MaxCli respectivamente.

Pen_FObj_MaxPrf, Pen_FObj_MinOcio: Desempeño unitario basados en sus valores óptimos para los objetivos FObj_MaxPrf y FObj_MinOcio.

\section{Variables de decisión}

$Y_{\text {idq: }} 1$ si se programa el paciente i el día d en el quirófano q, 0 de lo contrario.

$X_{\text {mdij: }} 1$ Si la cirugía del paciente i precede la cirugía del paciente j en el médico $m$ en el día d, 0 de lo contrario.

$\mathrm{G}_{\mathrm{qdij}}: 1 \mathrm{Si}$ la cirugía del paciente i precede la cirugía del paciente j en el quirófano q en el día d, 0 de lo contrario.

s1, e1: Momento de inicio y finalización de la cirugía del paciente i respectivamente.

$A_{m d}: 1$ Si se programa el médico $m$ en el día d, 0 de lo contrario.

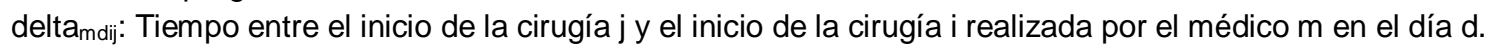

tar_pon: prioridad promedio de las cirugías asignadas.

num_med: Número de días médicos totales asignados para atender la demanda de pacientes.

num_cli: Número de clientes/pacientes totales asignados a cirugía.

FObj_MaxPrf: Preferencia total normalizada en escala de 1 a 0.

FObj_MinOcio: Tiempos ocioso total normalizado en escala de 1 a 0

OBC: Variable que toma el menor valor con respecto a las funciones objetivo

\section{REFERENCIAS}

Abdelaziz, F.B., Belaid, A., y Fayedh, R.E., Multi-Objective Stochastic Programming for Portfolio Selection, https://doi.org/10.1016/j.ejor.2005.10.021, European Journal of Operational Research, 177(3), 1811-1823 (2007).

Akbarzadeh, B., Moslehi, G., y otros dos autores, The Re-Planning and Scheduling of Surgical Cases in the Operating Room Department after Block Release Time with Resource Rescheduling, https://doi.org/10.1016/j.ejor.2019.04.037, European Journal of Operational Research, 278(2), 596-614 (2019).

Ali, H.H., Lamsali, H., y Othman, S.N., Operating Rooms Scheduling for Elective Surgeries in a Hospital Affected by WarRelated Incidents, https://doi.org/10.1007/s10916-019-1263-z, Journal of Medical Systems, 43(5) (2019).

Allouche, M.A., y otros cuatro autores, Solving Multi-Criteria Scheduling Flow Shop Problem through Compromise Programming and Satisfaction Functions, https://doi.org/10.1016/j.ejor.2007.09.038, European Journal of Operational Research, 192(2), 460-67 (2009).

Amiri, M., Ekhtiari, M., y Yazdani, M., Nadir Compromise Programming: A Model for Optimization of Multi-Objective Portfolio Problem, https://doi.org/10.1016/j.eswa.2010.12.061, Expert Systems with Applications, 38(6), 7222-7226 (2011).

Aranda, J., y Orjuela, J., Optimización Multiobjetivo en la Gestión de Cadenas de Suministro de Biocombustibles: Una Revisión de la Literatura, https://doi.org/10.14483/udistrital.jour.reving.2015.1.a03, Ingeniería, 20(1), 21-47 (2015).

Bovim, T., Christiansen, M., y otros tres autores, Stochastic Máster Surgery Scheduling,

https://doi.org/10.1016/j.ejor.2020.02.001, European Journal of Operational Research, 285(2), 695-711 (2020). 
Canales, F.A., Jurasz, J., y otros dos autores, Assessing Temporal Complementarity between Three Variable Energy Sources through Correlation and Compromise Programming, https://doi.org/10.1016/j.energy.2019.116637, Energy, 192, 116637 (2020).

Conforti, D., Guerriero, F., y Guido, R., A Multi-Objective Block Scheduling Model for the Management of Surgical Operating Rooms: New Solution Approaches via Genetic Algorithms, https://doi.org/10.1109/WHCM.2010.5441264, 2010 IEEE Workshop on Health Care Management, WHCM 2010, 1-5 (2010).

De la Hoz, E., Vélez, J., y López, L., Modelo de Programación Lineal Multiobjetivo para la Logística Inversa en el Sector Plástico de Polipropileno, https://dx.doi.org/10.4067/S0718-07642017000500005, Información Tecnológica, 28(5), 31-36 (2017)

Del Lapo-Maza, M. C., y Bustamante-Ubilla, M. A., Incidencia del Clima Organizacional y de las Actitudes Laborales en el Comportamiento Prosocial de los Profesionales de la Salud del Guayas Ecuador, DOI:10.4067/S071807642018000500245, Información Tecnológica, 29(5), 245-258 (2018)

Erekat, A., Servis, G., y otros dos autores, Efficient operating room planning using an ensemble learning approach to predict surgery cancellations, https://doi.org/10.1080/24725579.2019.1641576, IISE Transactions on Healthcare Systems Engineering, 10(1), 18-32 (2019).

Guido, R., y Conforti, D., A Hybrid Genetic Approach for Solving an Integrated Multi-Objective Operating Room Planning and Scheduling Problem, https://doi.org/10.1016/j.cor.2016.11.009, Computers and Operations Research, 87, 270-82, (2017).

Gür, Ş., Eren, T., y Alakaş H.M., Surgical Operation Scheduling with Goal Programming and Constraint Programming: A Case Study, https://doi.org/10.3390/math7030251, Mathematics 7 (3), 251 (2019).

Ibarra-Taquez, H. N., Dobrosz-Gómez, I., y Gómez, Miguel-Ángel, Optimización Multiobjetivo del Proceso Fenton en el Tratamiento de Aguas Residuales provenientes de la Producción de Café Soluble, http://dx.doi.org/10.4067/S071807642018000500111, Información Tecnológica, 29(5), 111-122 (2018).

Khodaei, H., Hajiali, M., y otros tres autores, Fuzzy-Based Heat and Power Hub Models for Cost-Emission Operation of an Industrial Consumer Using Compromise Programming, https://doi.org/10.1016/j.applthermaleng.2018.04.008, Applied Thermal Engineering, 137(December 2017), 395-405 (2018).

Londoño, M., Orejuela, J., y Gil, C., Programación de Cirugías Multi- Objetivo Considerando Niveles de Prioridad, Espacios, 41(03), 30-45 (2019).

Miao, P., Yue, Z., y otros tres autores, Optimal Emission Management of Photovoltaic and Wind Generation Based Energy Hub System Using Compromise Programming, https://doi.org/10.1016/j.jclepro.2020.124333, Journal of Cleaner Production, 281, 124333 (2020).

Milina, L., y Rivera, M., Percepción del Cliente Interno y Externo Sobre la Calidad de los Servicios en el Hospital General de Cárdenas Tabasco 2011, Salud en Tabasco, ISSN: 1405-2091,18(2), 56-63 (2011).

Ministerio de Salud, Informe Nacional de Calidad de la Atención en Salud, Technical Report, Bogotá (2015).

Ribeiro, J.F.F., Leoneti, A.B., y Costa, A.L., A Two-Phase Method for Operating Room Scheduling, https://doi.org/10.14488/BJOPM.2018.v15.n4.a1, Brazilian Journal of Operations \& Production Management, 15(4), 47180 (2018).

Saadouli, H., Jerbi, B., y otros tres autores, A Stochastic Optimization and Simulation Approach for Scheduling Operating Rooms and Recovery Beds in an Orthopedic Surgery Department, https://doi.org/10.1016/j.cie.2014.11.021, Computers and Industrial Engineering, 80, 72-79 (2015).

Samudra, M., Van Riet, C., y otros cuatro autores, Scheduling operating rooms: achievements, challenges and pitfalls, Journal of Scheduling, 19(5), 493-525 (2016).

Sipper, D., y Bulfin, R.L., Planeación y Control de la Producción, 415, McGraw-Hill, México (1998).

Velásquez-Restrepo, P. A., Rodríguez-Quintero, A. K., y Jaén-Posada, J. S., Aproximación Metodológica a la Planificación y a la Programación de las Salas de Cirugía: Una Revisión de la Literatura, https://doi.org/10.11144/Javeriana.rgsp12-24.ampp, Revista Gerencia y Políticas de Salud, 12(24), 249-66 (2013).

Torres Sanchez, M. J., y Estupiñan Gonzalez, A. M., Análisis de variabilidad en la programación de cirugías en un hospital de nivel iv en la ciudad de Bogotá, Applied Microbiology and Biotechnology, 85(1):2071-2079 (2014).

Younespour, M., Atighehchian, A., y otros dos autores, Using Mixed Integer Programming and Constraint Programming for Operating Rooms Scheduling with Modified Block Strategy, https://doi.org/10.1016/j.orhc.2019.100220, Operations Research for Health Care, 23,100220 (2019).

Zelany, M., A Concept of Compromise Solutions and the Method of the Displaced Ideal, https://doi.org/10.1016/03050548(74)90064-1, Computers \& Operations Research, 1(3-4), 479-496 (1974).

Zhang, Y., Wang, Y., y otros dos autores, Mitigating Overtime Risk in Tactical Surgical Scheduling, https://doi.org/10.1016/j.omega.2019.01.002, Omega (United Kingdom), 98, 1-13 (2019).

Zhu, S., Fan, W., y otros tres autores, Operating Room Planning and Surgical Case Scheduling: A Review of Literature, 10.1007/s10878-018-0322-6, Journal of Combinatorial Optimization, 37(3), 757-805 (2019). 
